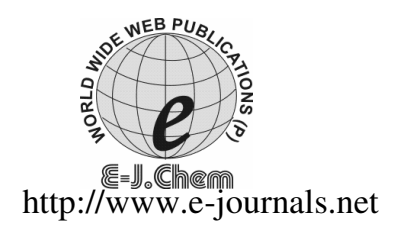

ISSN: 0973-4945; CODEN ECJHAO

E-Journal of Chemistry

2011, 8(2), 541-546

\title{
Synthesis and Biological Evaluation of Some New Chalcones Containing 2,5-Dimethylfuran Moiety
}

\author{
S.SRIDHAR ${ }^{*}$, S.C.DINDA ${ }^{\S}$ and Y.RAJENDRA PRASAD \\ * Chebrolu Hanumaiah Institute of Pharmaceutical Sciences \\ Guntur, A.P., India \\ ${ }^{\S}$ School of Pharmaceutical Education and Research \\ Berhampur University, Berhampur, Orissa, India \\ University College of Pharmaceutical Sciences \\ Andhra University, Vishakapatanam, A.P., India \\ sridhar_pharma24@yahoo.co.in
}

Received 21 June 2010; Accepted 2 September 2010

\begin{abstract}
A series of new chalcones (3a-g) were prepared by Claisen-Schmidt condensation of 3-acetyl-2,5-dimethylfuran with various substituted aromatic aldehydes in presence of aqueous solution of potassium hydroxide and ethanol at room temperature. The synthesized chalcones were characterized by means of their IR, ${ }^{1} \mathrm{H}$ NMR spectral data and elemental analyses. When these chalcones were evaluated for antimicrobial and anti-inflammatory activities, some of them were found to possess significant activity, when compared to standard drugs.
\end{abstract}

Keywords: Chalcone, Antimicrobial activity, Synthesis, Dimethylfuran moiety.

\section{Introduction}

The search for new potent anti-microbial agents with reduced toxicity and lower side effects is of continuous process. One of the most frequently encountered groups of organic compounds in medicinal chemistry is chalcones and their derivatives. Chalcone is a generic term given to compounds bearing the 1,3-diphenylprop-2-en-1-one frame work, which can be functionalized in the propane chain by the presence olefinic, keto and/or hydroxyl groups (Figure 1$)^{1}$. Their bactericidal effect has been related to the ability of the $\alpha, \beta$ - unsaturated ketone to undergo a conjugated addition to a nucleophilic group like a thiol group in an essential protein. In addition, chalcone derivatives showed activity against dermatophytes only but not against other types of fungi. Chalcones are readily synthesized by the base catalysed Claisen-Schmidt condensation of an aldehyde and an appropriate ketone in a polar solvent like ethanol and yields may be variable, ranging ${ }^{2-3}$ from $5 \%$ to $80 \%$. The chalcones have a diverse range of biological activities, among which antimalarial, antitubercular, antiinflammatory, cytotoxic, antioxidant, analgesic, antiviral and antimicrobial ${ }^{4-10}$ properties were widely cited. 
<smiles>C=C(C=Cc1ccccc1)c1ccccc1</smiles>

Figure 1

As shown in scheme 1 , chalcones $\mathbf{3}(\mathbf{a}-\mathbf{g})$ were synthesized by a base catalyzed condensation of 3-acetyl-2,5-dimethylfuran and appropriately substituted aldehydes ${ }^{11}$. The structures of various synthesized compounds were characterized on the basis of elemental analyses, IR and ${ }^{1} \mathrm{H}$ NMR spectral data. The compounds were evaluated for their antiinflammatory activity by carrageenan induced rat paw oedema method and antimicrobial activity by agar cup plate method.

\section{Experimental}

Melting points were determined on an open capillary melting point apparatus and are uncorrected. ${ }^{1} \mathrm{H}$ NMR spectra were recorded in $\mathrm{CDCl}_{3}$ on Bruker WM $400 \mathrm{MHz}$ spectrometer with TMS as internal standard. Infrared spectra were recorded $(\mathrm{KBr})$ on a Perkin-Elmer AC-1 spectrophotometer. Micro analyses were performed on Carlo Erba E-1108 element analyzer and were with in the $\pm 0.4 \%$ of the theoretical values. Reaction completion was identified by TLC using silica gel for TLC (Merck). All the chalcones have been purified by column chromatography performed on silica gel columns (100-200 mesh, Merck).

General procedure for the synthesis of 1-(2',5'-dimethyl-3'-furyl)-3-(aryl)-2-propen1- one (3a-g)

A mixture of 3-acetyl-2,5-dimethylfuran $(0.005 \mathrm{~mol})(\mathbf{1})$ and respective aldehyde $(0.005 \mathrm{~mol})$ (2) was stirred in ethanol $(7.5 \mathrm{~mL})$ and then an aqueous solution of potassium hydroxide $(50 \%$, $7.5 \mathrm{~mL}$ ) was added to it. The mixture was kept for $24 \mathrm{~h}$ and it was acidified with $1: 1 \mathrm{HCl}$ and $\mathrm{H}_{2} \mathrm{O}$. Then it was filtered under vacuum and the solid was washed with water, purified by column chromatography and crystallized from a mixture of ethyl acetate and hexane (Scheme 1). The physical and spectral data of the chalcones were shown in Table $1 \& 2$.<smiles>CCCC(=O)c1cc(C)oc1C</smiles>

Where $\mathrm{Ar}=$

Scheme 1. Synthesis of chalcones<smiles>COc1cc(C)cc(OC)c1OC</smiles>

$3 a$

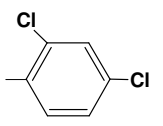

$3 \mathbf{e}$

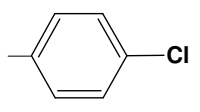

3b

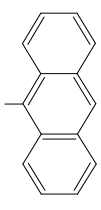

3f

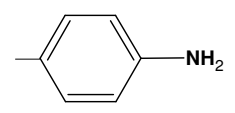

$3 c$

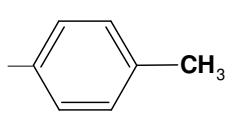

3d

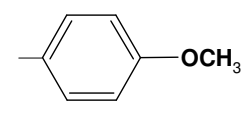

$3 g$ 
Table 1. Physical data of the prepared compounds $3(\mathbf{a}-\mathbf{g})$

\begin{tabular}{|c|c|c|c|c|c|c|c|c|c|c|c|}
\hline \multirow{2}{*}{ S.No. } & \multirow{2}{*}{$\mathrm{Ar}$} & \multirow{2}{*}{$\begin{array}{l}\text { Mol. } \\
\text { Formula }\end{array}$} & \multirow{2}{*}{ 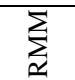 } & \multirow{2}{*}{ U } & \multirow{2}{*}{$\stackrel{2}{2} \stackrel{0}{\circ}$} & \multicolumn{3}{|c|}{$\%$ Calc. } & \multicolumn{3}{|c|}{$\%$ Found } \\
\hline & & & & & & $\mathrm{C}$ & $\mathrm{H}$ & $\mathrm{N}$ & $\mathrm{C}$ & $\mathrm{H}$ & $\mathrm{N}$ \\
\hline $\mathbf{3 a}$ & $\begin{array}{l}3 ", 4 ", 5 "- \\
\text { trimethoxyphenyl }\end{array}$ & $\mathrm{C}_{18} \mathrm{H}_{20} \mathrm{O}_{5}$ & 316 & 154 & 71 & 68.34 & 6.37 & - & 68.23 & 6.28 & - \\
\hline 3b & 4"-chlorophenyl & $\mathrm{C}_{15} \mathrm{H}_{13} \mathrm{ClO}_{2}$ & 260 & 139 & 68 & 69.23 & 5.01 & - & 69.25 & 5.03 & - \\
\hline $3 c$ & 4"-dimethylaminophenyl & $\mathrm{C}_{17} \mathrm{H}_{19} \mathrm{NO}_{2}$ & 269 & 112 & 73 & 75.83 & 7.06 & 5.20 & 75.82 & 7.05 & 5.22 \\
\hline 3d & 4"-methylphenyl & $\mathrm{C}_{16} \mathrm{H}_{16} \mathrm{O}_{2}$ & 240 & 153 & 75 & 80.02 & 6.66 & - & 80.04 & 6.68 & - \\
\hline $3 e$ & $2^{\prime \prime}, 4^{\prime \prime}$-dichlorophenyl & $\mathrm{C}_{15} \mathrm{H}_{12} \mathrm{Cl}_{2} \mathrm{O}_{2}$ & 294 & 132 & 71 & 61.22 & 4.08 & - & 61.24 & 4.06 & - \\
\hline 3f & 9"-anthracenyl & $\mathrm{C}_{23} \mathrm{H}_{18} \mathrm{O}_{2}$ & 326 & 138 & 78 & 84.64 & 5.56 & - & 84.65 & 5.58 & - \\
\hline $3 \mathrm{~g}$ & 4"-methoxyphenyl & $\mathrm{C}_{16} \mathrm{H}_{16} \mathrm{O}_{3}$ & 256 & 87 & 73 & 75.01 & 6.25 & - & 75.02 & 6.28 & - \\
\hline
\end{tabular}

Table 2. Spectral data of the prepared compounds 3(a-g)

\begin{tabular}{cl}
\hline S.No & \multicolumn{1}{c}{ IR, v $v_{\max }, \mathrm{cm}^{-1}$} \\
\hline 3a & $1655(\mathrm{C}=\mathrm{O}), 1602(\mathrm{C}=\mathrm{C}$ of \\
& $\mathrm{Ar}), 1505(\mathrm{CH}=\mathrm{CH}), 1125$ \\
& $\left(\mathrm{O}-\mathrm{CH}_{3}\right), 1048(\mathrm{C}-\mathrm{O}-\mathrm{C})$ \\
& \\
3b & $1664(\mathrm{C}=\mathrm{O}), 1584(\mathrm{C}=\mathrm{C}$ of \\
& Ar $), 1520(\mathrm{CH}=\mathrm{CH}), 1060$ \\
& $(\mathrm{C}-\mathrm{O}), 855(\mathrm{C}-\mathrm{Cl})$
\end{tabular}

3c $\quad 1658(\mathrm{C}=\mathrm{O}), 1588(\mathrm{C}=\mathrm{C}$ of $\mathrm{Ar}), 1502(\mathrm{CH}=\mathrm{CH}), 1185$ $\left(-\mathrm{N}\left(\mathrm{CH}_{3}\right)_{2}\right) 1082(\mathrm{C}-\mathrm{O})$

3.90-3.95 (9H, s, 3x-OCH 3$), 6.71(1 \mathrm{H}, \mathrm{s}, \mathrm{C}-4$ '$\mathrm{H}), 7.20(1 \mathrm{H}, \mathrm{d}, \mathrm{J}=17 \mathrm{~Hz},-\mathrm{CO}-\mathrm{CH}=), 7.81(1 \mathrm{H}$, $\mathrm{d}, \mathrm{J}=17 \mathrm{~Hz},=\mathrm{CH}-\mathrm{Ar}), 6.90\left(2 \mathrm{H}, \mathrm{s}, \mathrm{C}-2^{\prime \prime}-\mathrm{H}\right.$ and C-6"-H), 2.2(3H,s, Ar- $\left.\mathrm{CH}_{3}\right), 2.7\left(3 \mathrm{H}, \mathrm{s}, \mathrm{Ar}-\mathrm{CH}_{3}\right)$. $6.41(1 \mathrm{H}, \mathrm{s}, \mathrm{C}-4 \mathrm{\prime}-\mathrm{H}), 7.10(1 \mathrm{H}, \mathrm{d}, \mathrm{J}=17 \mathrm{~Hz},-\mathrm{CO}-$ $\mathrm{CH}=), 7.70(1 \mathrm{H}, \mathrm{d}, \mathrm{J}=17 \mathrm{~Hz},=\mathrm{CH}-\mathrm{Ar}), 7.58(2 \mathrm{H}$, $\mathrm{d}, \mathrm{J}=8 \mathrm{~Hz}, \mathrm{C}-3^{\prime \prime}-\mathrm{H}$ and $\left.\mathrm{C}-5^{\prime \prime}-\mathrm{H}\right), 7.40(2 \mathrm{H}, \mathrm{d}, \mathrm{J}=$ $8 \mathrm{~Hz}, \mathrm{C}-2^{\prime \prime}-\mathrm{H}$ and $\left.\mathrm{C}-6^{\prime \prime}-\mathrm{H}\right), 2.3(3 \mathrm{H}, \mathrm{s}, \mathrm{Ar}-$ $\left.\mathrm{CH}_{3}\right), 2.6\left(3 \mathrm{H}, \mathrm{s}, \mathrm{Ar}-\mathrm{CH}_{3}\right)$.

$3.05\left(6 \mathrm{H}, \mathrm{s},-\mathrm{N}\left(\mathrm{CH}_{3}\right)_{2}\right), 6.58\left(1 \mathrm{H}, \mathrm{s}, \mathrm{C}-4{ }^{\prime}-\mathrm{H}\right), 7.25$ $(1 \mathrm{H}, \mathrm{d}, \mathrm{J}=17 \mathrm{~Hz},-\mathrm{CO}-\mathrm{CH}=), 7.87(1 \mathrm{H}, \mathrm{d}, \mathrm{J}=17 \mathrm{~Hz}$, $-\mathrm{CH}=\mathrm{Ar}), 6.75\left(2 \mathrm{H}, \mathrm{d}, \mathrm{J}=8.5 \mathrm{~Hz}, \mathrm{C}-2^{\prime \prime}-\mathrm{H}\right.$ and C-6"$\mathrm{H}), 7.55\left(2 \mathrm{H}, \mathrm{d}, \mathrm{J}=8.5 \mathrm{~Hz}, \mathrm{C}-3^{\prime \prime}-\mathrm{H}\right.$ andC-5"-H), $2.05\left(3 \mathrm{H}, \mathrm{s}, \mathrm{Ar}-\mathrm{CH}_{3}\right), 2.5\left(3 \mathrm{H}, \mathrm{s}, \mathrm{Ar}-\mathrm{CH}_{3}\right)$.

3d $1641(\mathrm{C}=\mathrm{O}), 1583(\mathrm{C}=\mathrm{C}$ of $2.60\left(3 \mathrm{H}, \mathrm{s}, \mathrm{Ar}-\mathrm{CH}_{3}\right), 6.30(1 \mathrm{H}, \mathrm{s}, \mathrm{C}-4 \mathrm{-}-\mathrm{H}), 7.23$ $\mathrm{Ar}), 1505(\mathrm{CH}=\mathrm{CH}), 1076$ (C-O) $(1 \mathrm{H}, \mathrm{d}, \mathrm{J}=17 \mathrm{~Hz},-\mathrm{CO}-\mathrm{CH}=), 7.77(1 \mathrm{H}, \mathrm{d}, \mathrm{J}=$ $17 \mathrm{~Hz},-\mathrm{CH}=\mathrm{Ar}), 7.56\left(2 \mathrm{H}, \mathrm{d}, \mathrm{J}=9 \mathrm{~Hz}, \mathrm{C}-3^{\prime \prime}-\mathrm{H}\right.$ and $\left.\mathrm{C}-5^{\prime \prime} \mathrm{H}\right), 7.3\left(2 \mathrm{H}, \mathrm{d}, \mathrm{J}=9 \mathrm{~Hz}, \mathrm{C}-2^{\prime \prime}-\mathrm{H}\right.$ and C-6"$\mathrm{H}), 2.3\left(3 \mathrm{H}, \mathrm{s}, \mathrm{Ar}-\mathrm{CH}_{3}\right), 2.4\left(3 \mathrm{H}, \mathrm{s}, \mathrm{Ar}-\mathrm{CH}_{3}\right)$.

3e $\quad 1648(\mathrm{C}=\mathrm{O}), 1609(\mathrm{C}=\mathrm{C}$ of $6.3(1 \mathrm{H}, \mathrm{s}, \mathrm{C}-4$ '-H), $7.1(1 \mathrm{H}, \mathrm{d}, \mathrm{J}=17 \mathrm{~Hz},-\mathrm{CO}-\mathrm{CH}=)$, $\mathrm{Ar}), 1510(\mathrm{CH}=\mathrm{CH}), 1080$ (C-O), $868(\mathrm{C}-\mathrm{Cl})$ $7.99(1 \mathrm{H}, \mathrm{d}, \mathrm{J}=17 \mathrm{~Hz},=\mathrm{CH}-\mathrm{Ar}), 7.3(1 \mathrm{H}, \mathrm{d}, \mathrm{J}=8 \mathrm{~Hz}$, C-6"-H), 7.4(1H,s,C-3"-H), 7.6(1H,d, J=9HZ, C-5"-H), 2.3(3H,s, $\left.\mathrm{Ar}-\mathrm{CH}_{3}\right), 2.6\left(3 \mathrm{H}, \mathrm{s}, \mathrm{Ar}-\mathrm{CH}_{3}\right)$.

3f $1633(\mathrm{C}=\mathrm{O}), 1591(\mathrm{C}=\mathrm{C}$ of $6.20(1 \mathrm{H}, \mathrm{s}, \mathrm{C}-4$ '-H), $7.36(1 \mathrm{H}, \mathrm{d}, \mathrm{J}=17 \mathrm{~Hz},-\mathrm{CO}-$ $\mathrm{Ar}), 1506(\mathrm{CH}=\mathrm{CH}), 1070$ $\mathrm{CH}=), 7.68(1 \mathrm{H}, \mathrm{d}, \mathrm{J}=17 \mathrm{~Hz},=\mathrm{CH}-\mathrm{Ar}), 7.50-8.90$ (C-O)

$3 g \quad 1664(\mathrm{C}=\mathrm{O}), 1585(\mathrm{C}=\mathrm{C}$ of $(9 \mathrm{H}, \mathrm{Ar}-\mathrm{H}), 2.3\left(3 \mathrm{H}, \mathrm{s}, \mathrm{Ar}-\mathrm{CH}_{3}\right), 2.7\left(3 \mathrm{H}, \mathrm{s}, \mathrm{Ar}-\mathrm{CH}_{3}\right)$. $3.85\left(3 \mathrm{H}, \mathrm{s}, \mathrm{OCH}_{3}\right), 6.3\left(1 \mathrm{H}, \mathrm{s}, \mathrm{C}-4{ }^{\prime}-\mathrm{H}\right), 6.9(1 \mathrm{H}$, $\mathrm{Ar}), 1505(\mathrm{CH}=\mathrm{CH}), 1133$ $\left(\mathrm{O}-\mathrm{CH}_{3}\right), 1055(\mathrm{C}-\mathrm{O}-\mathrm{C})$ $\mathrm{d}, \mathrm{J}=17 \mathrm{~Hz},-\mathrm{CO}-\mathrm{CH}=)$, 7.1-7.6 (4H, m, C-2", 3", 5"and 6"-H), $7.7(1 \mathrm{H}, \mathrm{d}, \mathrm{J}=17 \mathrm{~Hz},=\mathrm{CH}-\mathrm{Ar})$, $2.3\left(3 \mathrm{H}, \mathrm{s}, \mathrm{Ar}-\mathrm{CH}_{3}\right), 2.5\left(3 \mathrm{H}, \mathrm{s}, \mathrm{Ar}-\mathrm{CH}_{3}\right)$.

Anti-inflammatory activity

Spraygue-dawley rats (M/S Gosh Enterprises, Calcutta, West Bengal, India) of either sex weighing between 180-200 g were used in the experiment. $1 \%$ carrageenan sodium gel was prepared with saline water for producing inflammation and gel of $1 \%$ sodium CMC was prepared with saline water for suspending the test compounds and standard drug. 
Rats were divided into nine groups of five animals each. Inflammation was induced by injecting $0.05 \mathrm{~mL}$ of $1 \%$ carrageenan subcutaneously into the sub plantar region of the right hind paw and $0.05 \mathrm{~mL}$ of saline was injected into the sub plantar region of the left hind paw for all groups. $1 \%$ sodium CMC gel $(1 \mathrm{~mL} / \mathrm{kg})$ was given to group-I used as carrageenan treated control and the standard drug aceclofenac $(2 \mathrm{mg} / \mathrm{kg})$ was administered to group-II. The groups III-IX treated with compounds 3(a-g) $(10 \mathrm{mg} / \mathrm{kg})$. All the doses were administered orally. Anti-inflammatory activity was evaluated by measuring carrageenan induced paw oedema ${ }^{12,13}$. The thickness of raw paw was measured before carrageenan injection and after carrageenan injection at time intervals $0.5,1,2,3,4$ and $6 \mathrm{~h}$ using Zeitlin constant loaded lever method ${ }^{14}$. The percentage increase of paw oedema thickness was calculated $^{15}$. The results and statistical analysis of anti-inflammatory activity of aceclofenac and the compounds tested were shown in Table 3.

Table 3. Anti-inflammatory activity of chalcones 3 (a-g)

\begin{tabular}{ccccccc}
\hline \multirow{2}{*}{ Compound } & \multicolumn{7}{c}{ \% inhibition \pm SEM at various time intervals } \\
\cline { 2 - 7 } & $0.5 \mathrm{~h}$ & $1.0 \mathrm{~h}$ & $2.0 \mathrm{~h}$ & $3.0 \mathrm{~h}$ & $4.0 \mathrm{~h}$ & $6.0 \mathrm{~h}$ \\
\hline $\mathbf{3 a}$ & $20.58 \pm 0.91$ & $31.65 \pm 1.01$ & $59.84 \pm 1.53$ & $69.13 \pm 1.25$ & $76.55 \pm 1.98$ & $85.15 \pm 2.22$ \\
3b & $24.19 \pm 0.78^{*}$ & $42.57 \pm 0.99^{*}$ & $65.47 \pm 1.65^{*}$ & $79.87 \pm 1.66^{*}$ & $89.28 \pm 2.01$ & $97.28 \pm 1.99$ \\
$\mathbf{3 c}$ & $21.10 \pm 0.65$ & $41.46 \pm 1.12^{*}$ & $53.13 \pm 1.36^{*}$ & $72.91 \pm 1.67$ & $82.65 \pm 2.32$ & $72.55 \pm 2.32$ \\
$\mathbf{3 d}$ & $17.13 \pm 0.75^{*}$ & $28.02 \pm 0.89$ & $42.37 \pm 1.45^{*}$ & $67.81 \pm 1.33^{*}$ & $78.74 \pm 1.88$ & $82.70 \pm 2.53$ \\
$\mathbf{3 e}$ & $24.78 \pm 0.77^{*}$ & $37.37 \pm 0.92^{*}$ & $51.11 \pm 1.35$ & $62.35 \pm 1.81^{*}$ & $84.10 \pm 2.95$ & $87.30 \pm 3.25$ \\
$\mathbf{3 f}$ & $23.01 \pm 0.65^{*}$ & $36.25 \pm 1.23^{*}$ & $57.10 \pm 1.48$ & $78.14 \pm 1.59$ & $80.10 \pm 2.32$ & $85.15 \pm 2.31$ \\
$\mathbf{3 g}$ & $21.68 \pm 0.88$ & $34.11 \pm 1.02^{*}$ & $57.45 \pm 1.43$ & $76.16 \pm 1.53$ & $82.46 \pm 1.95$ & $89.52 \pm 2.35$ \\
Aceclofenac & $28.0 \pm 2.0$ & $20.16 \pm 0.90$ & $43.85 \pm 0.97$ & $58.10 \pm 1.52$ & $77.83 \pm 1.68$ & $77.19 \pm 1.97$ \\
\hline All values are represented as mean \pm SEM $(n=6) .{ }^{*} P<0.01$ compared to reference standard Aceclofenac \\
Student's t-test. Dosage: Aceclofenac- $2 \mathrm{mg} / \mathrm{kg}$ and test compounds-10 mg/kg body weight of rat
\end{tabular}

Antimicrobial activity

Cup plate method ${ }^{16,17}$ using Mueller-Hinton agar medium was employed to study the preliminary antibacterial activity of (3a-g) against B. subtilis, B. pumilis, S. aureus, E. coli and $P$. vulgaris. The agar medium was purchased from HI-media Laboratories Ltd., Mumbai, India. Preparation of nutrient broth, subculture, base layer medium, agar medium and peptone water was done as per the standard procedure. Each test compound $(5 \mathrm{mg})$ was dissolved in $5 \mathrm{~mL}$ of dimethyl sulfoxide. Benzylpenicillin was employed as reference standard $(1000 \mu \mathrm{g} / \mathrm{mL})$ to compare the results. The $\mathrm{pH}$ of the all the test solutions and control was maintained at 2-3 by using conc. $\mathrm{HCl}$, because the compounds were not diffused through agar medium at $\mathrm{pH}$ below 3. All the compounds were tested at a concentration of $0.05 \mathrm{~mL}(50 \mu \mathrm{g})$ and $0.1 \mathrm{~mL}(100 \mu \mathrm{g})$ level and DMSO as control did not show any inhibition.

Same cup plate method using PDA (Potato-Dextrose-Agar) medium was employed to study the preliminary antifungal activity of (3a-g) against A. niger, C. albicans and $R$. oryzae. The PDA medium was purchased from HI-media Laboratories Ltd., Mumbai, India. Preparation of nutrient broth, subculture, base layer medium and PDA medium was done as per the standard procedure. Each test compound $(5 \mathrm{mg})$ was dissolved in $5 \mathrm{~mL}$ of dimethyl sulfoxide. Fluconazole employed as reference standard $(1000 \mu \mathrm{g} / \mathrm{mL})$ to compare the results. The $\mathrm{pH}$ of the all the test solutions and control was maintained at 2-3 by using conc. $\mathrm{HCl}$, because the compounds were not diffused through agar medium at $\mathrm{pH}$ below 3 . All the compounds were tested at a concentration of $0.05 \mathrm{~mL}(50 \mu \mathrm{g})$ and $0.1 \mathrm{~mL}(100 \mu \mathrm{g})$ level and DMSO as control did not show any inhibition.

The cups each of $7 \mathrm{~mm}$ diameter were made by scooping out medium with a sterilized cork borer in a Petri dish which was streaked with the organisms. The solutions of each test 
compound, control and reference standards $(0.05$ and $0.1 \mathrm{~mL})$ were added separately in the cups and Petri dishes were subsequently incubated at $37 \pm 1{ }^{\circ} \mathrm{C}$ for $24 \mathrm{~h}$ for antibacterial activity and kept aside at room temperature for $48 \mathrm{~h}$ for antifungal activity. Zone of inhibition produced by each compound was measured in $\mathrm{mm}$ and the results are presented in Table. 4 for antibacterial activity and in Table.5 for antifungal activity.

Table 4. Antibacterial activity of chalcones $\mathbf{3}(\mathbf{a}-\mathbf{g})$

\begin{tabular}{|c|c|c|c|c|c|c|c|c|c|}
\hline \multirow{3}{*}{ Compound } & \multicolumn{9}{|c|}{ Zone of inhibition, in $\mathrm{mm}$} \\
\hline & \multicolumn{9}{|c|}{ Quantity in $\mu \mathrm{g} / \mathrm{mL}$} \\
\hline & 50 & 100 & 50 & 100 & 50 & 100 & 50 & 100 & 50 \\
\hline $3 \mathbf{a}$ & 12 & 11 & 09 & 10 & 08 & 11 & 11 & 12 & 13 \\
\hline $3 \mathbf{b}$ & 15 & 21 & 17 & 20 & 18 & 23 & 14 & 15 & 14 \\
\hline $3 c$ & 16 & 18 & 18 & 23 & 20 & 23 & 18 & 23 & 21 \\
\hline 3d & 20 & 23 & 19 & 16 & 11 & 19 & 18 & 19 & 17 \\
\hline $3 e$ & 11 & 13 & 14 & 14 & 17 & 21 & 12 & 16 & 11 \\
\hline 3f & 14 & 16 & 18 & 23 & 14 & 21 & 15 & 17 & 16 \\
\hline 3g & 19 & 22 & 20 & 24 & 18 & 22 & 16 & 17 & 13 \\
\hline Benzylpenicillin & 27 & 32 & 30 & 31 & 26 & 29 & 24 & 26 & 27 \\
\hline \multirow[t]{2}{*}{ Control } & - & - & - & - & - & - & - & - & - \\
\hline & Tabl & 5. $\mathrm{An}$ & tifung & activi & y of & halcon & $3(\mathbf{a}-\xi$ & & \\
\hline \multirow{4}{*}{ Compound } & \multicolumn{9}{|c|}{ Zone of inhibition, in $\mathrm{mm}$} \\
\hline & \multicolumn{9}{|c|}{ Quantity in $\mu \mathrm{g} / \mathrm{mL}$} \\
\hline & \multicolumn{3}{|c|}{ A. niger } & \multicolumn{3}{|c|}{ C. albicans } & \multicolumn{3}{|c|}{ R. oryzae } \\
\hline & & 0 & 100 & 50 & & 100 & 50 & & 100 \\
\hline $3 \mathbf{a}$ & & 6 & 20 & 17 & & 21 & 17 & & 19 \\
\hline $3 \mathbf{b}$ & & 7 & 21 & 15 & & 20 & 15 & & 18 \\
\hline $3 c$ & & 7 & 23 & 24 & & 25 & 16 & & 18 \\
\hline 3d & & 4 & 17 & 16 & & 21 & 13 & & 18 \\
\hline $3 e$ & & 5 & 17 & 20 & & 22 & 13 & & 16 \\
\hline $3 f$ & & 8 & 20 & 22 & & 20 & 14 & & 19 \\
\hline 3g & & 7 & 20 & 21 & & 22 & 15 & & 18 \\
\hline Fluconazole & & 3 & 27 & 23 & & 27 & 21 & & 26 \\
\hline Control & & - & - & - & & - & - & & - \\
\hline
\end{tabular}

\section{Results and Discussion}

The results of anti-inflammatory activity revealed that the compounds (3a-g) exhibited moderate to considerable activity when compared to reference standard aceclofenac. In addition, it was found that $\mathbf{3 b}$ showed maximum activity and this may be due to presence of chloro group at $4^{\text {th }}$ position on aromatic ring-B of chalcone. Moreover, it was also observed that the compounds $\mathbf{3 e}$ and $\mathbf{3 g}$, carrying 2,4-dichlorophenyl and 4-methoxy phenyl rings as ring-B of chalcone, respectively, showed remarkable activity.

Compounds (3a-g) showed significant antibacterial activity at both $0.05 \mathrm{~mL}(50 \mu \mathrm{g})$ and $0.1 \mathrm{~mL}(100 \mu \mathrm{g})$ concentration levels when compared with standard drug benzylpenicillin. However, the chalcones $\mathbf{3 b}, \mathbf{3 c}$ and $\mathbf{3 g}$ were found to be more potent on all the bacterial strains. Compounds (3a-g) also showed significant antifungal activity at both $0.05 \mathrm{~mL}(50 \mu \mathrm{g})$ and $0.1 \mathrm{~mL}(100 \mu \mathrm{g})$ concentration levels when compared with standard drug fluconazole. Compounds $\mathbf{3 c}$ and $\mathbf{3 g}$ showed maximal antifungal activity. The results clearly revealed the contribution of electron withdrawing groups and electron releasing groups on the aromatic ring in enhancing the antibacterial and antifungal activity. 


\section{Acknowledgment}

The authors are thankful to Dr. Trimurtulu, Vice-President, Laila Impex Industries Ltd., Vijayawada, India for providing IR, ${ }^{1} \mathrm{H}$ NMR and elemental analyses data.

\section{References}

1. Oyvind $\mathrm{M}$ A and Kenneth R M, Flavonoids: Chemistry, Biochemistry and Applications, CRC Press: New York, 2006, 1004.

2. Prasad Y R, Ravikumar P, ASHA Deepti CH and Ramana M V, Asian J Chem., 2007, 19(6), 4799-4804.

3. Prasad Y R, Ravikumar P, Srivani N and Rao A S, Int J Chem Sci., 2006, 4(4), 905-909.

4. Tomar V, Bhattacharjee G, Kamaluddin and Kumar A, Bioorg Med Chem Lett., 2007, 17(19), 5321-5324.

5. Solankee A, Kapadia K, Ciric A, Sokovic M, Doytchinova I and Geronikaki A, Eur J. Med Chem., 2010, 45(2), 510-518.

6. Mei L, Prapon W, Simon L C, Agnes L-C T and Mei-Lin G, Bioorg Med Chem., 2003, 11(13), 2729-2738.

7. Trivedi A R, Dodiya D K, Ravat N R and Shah V H, ARKIVOC, 2008, xi, 131-141.

8. Jen-Hao C, Chi-Feng H, Shyh-Chyun Y, Jih-Pyang W, Shen-Jeu W and Chun-Nan L, Bioorg Med Chem., 2008, 16(15), 7270-7276.

9. Bhat B A, Dhar K L, Puri S C, Saxena A K, Shanmugavelb M and Qazi G N, Bioorg Med Chem Lett., 2005, 15, 3177.

10. Jalpa C T, Jitender B B, Kuldip D U, Yogesh T N, Sudhir K J, Christophe C P, Erik De C and Anamik K S, Tetrahedron Lett., 2007, 48(48), 8472-8474.

11. Dhar D N, The Chemistry of Chalcones and related compounds; John Wiley and Sons, New York, 1981.

12. Turner R A, Screening Methods in Pharmacology; Demic Press: New York, 1965, 152.

13. Winter C A, Risley E A and Nuss G W, Proc Soc Exp Biol Med., 1962, 111, 544-547.

14. Al-Haboubi H A and Zeitlin I J, Eur J Pharmacol., 1983, 88, 169-176.

15. Duwiejua M, Zeitlin I J, Watermanand P G and Gray A I, J Pharm Pharmacol., 1994, 46, 286-290.

16. Banty A L, The antimicrobial Susceptibility test: Principle and practice; Edited by Illus, Lea and Febiger, Philadelphia, PA: USA, 1976, 180.

17. Seely H W and Van Demark P J, Microbes in Action: A Laboratory Manual of Microbiology; D.B. Taraporewala Sons and Co., Bombay, 1975, 55. 


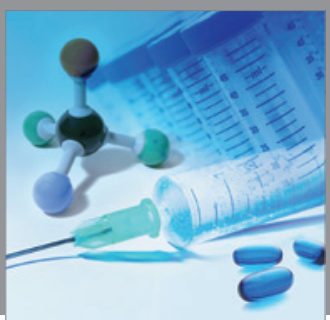

International Journal of

Medicinal Chemistry

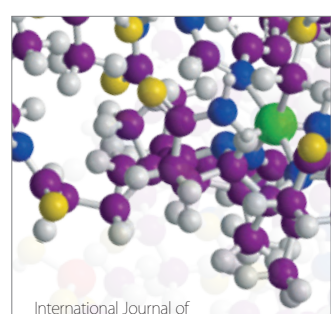

Carbohydrate Chemistry

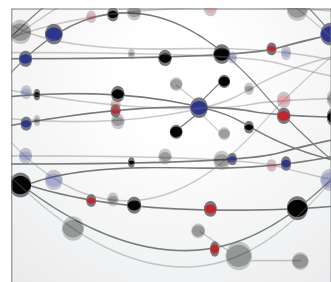

The Scientific World Journal
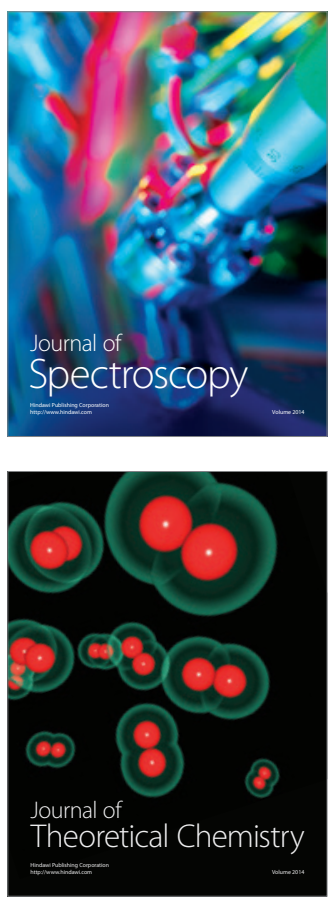
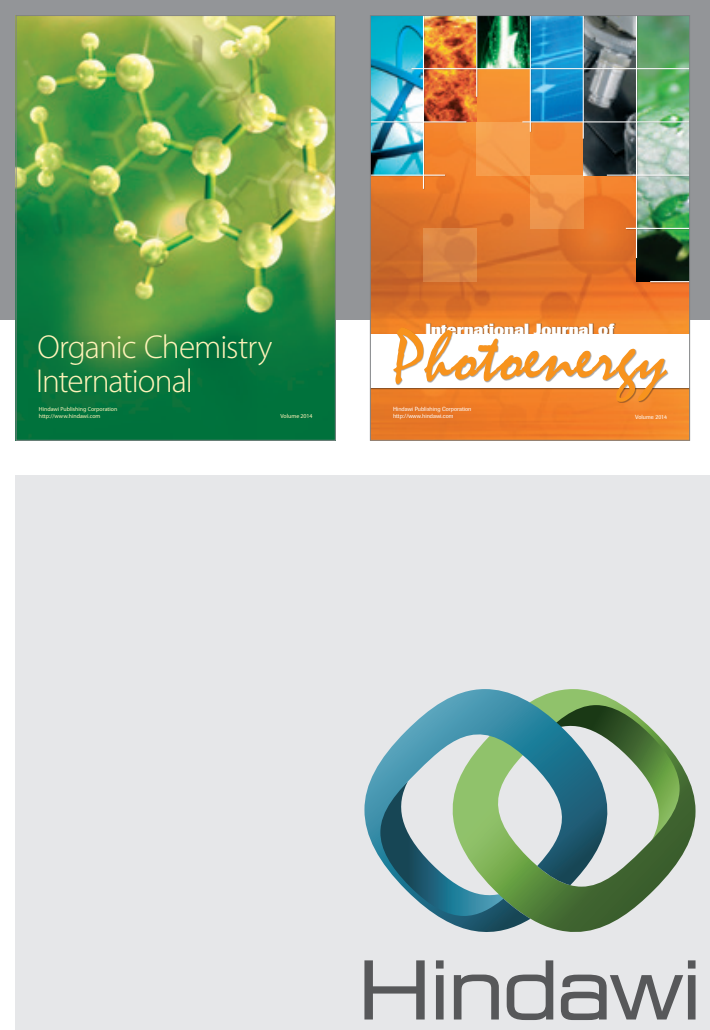

Submit your manuscripts at

http://www.hindawi.com
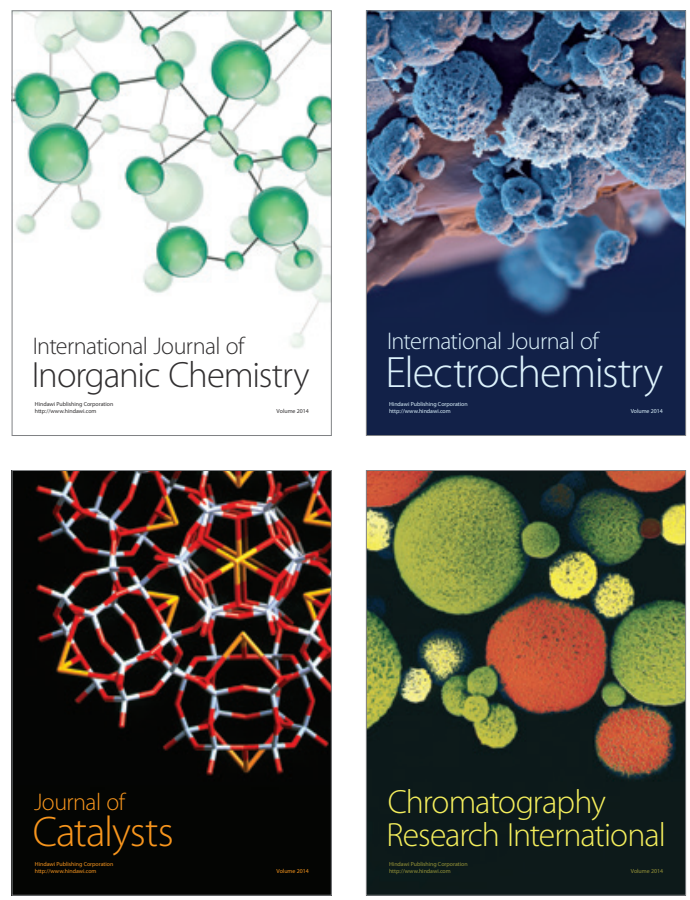
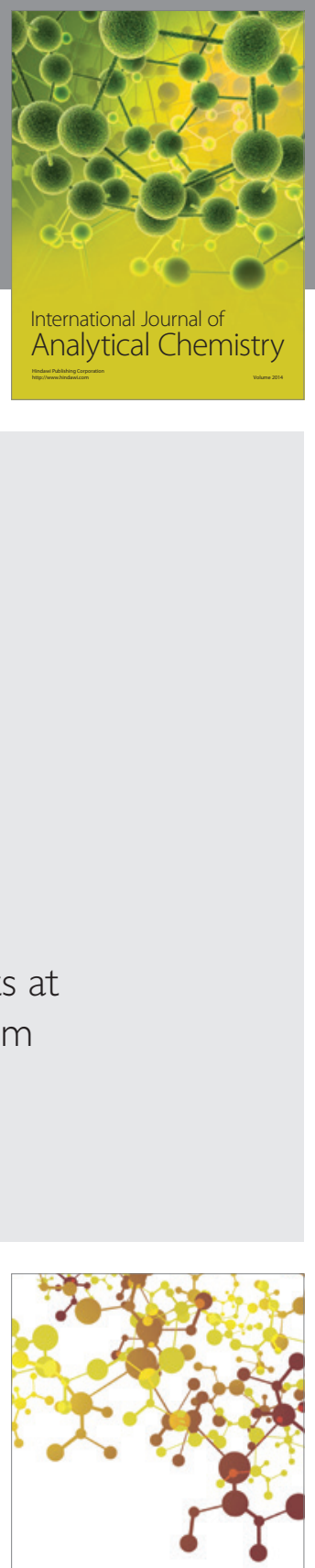

Journal of

Applied Chemistry
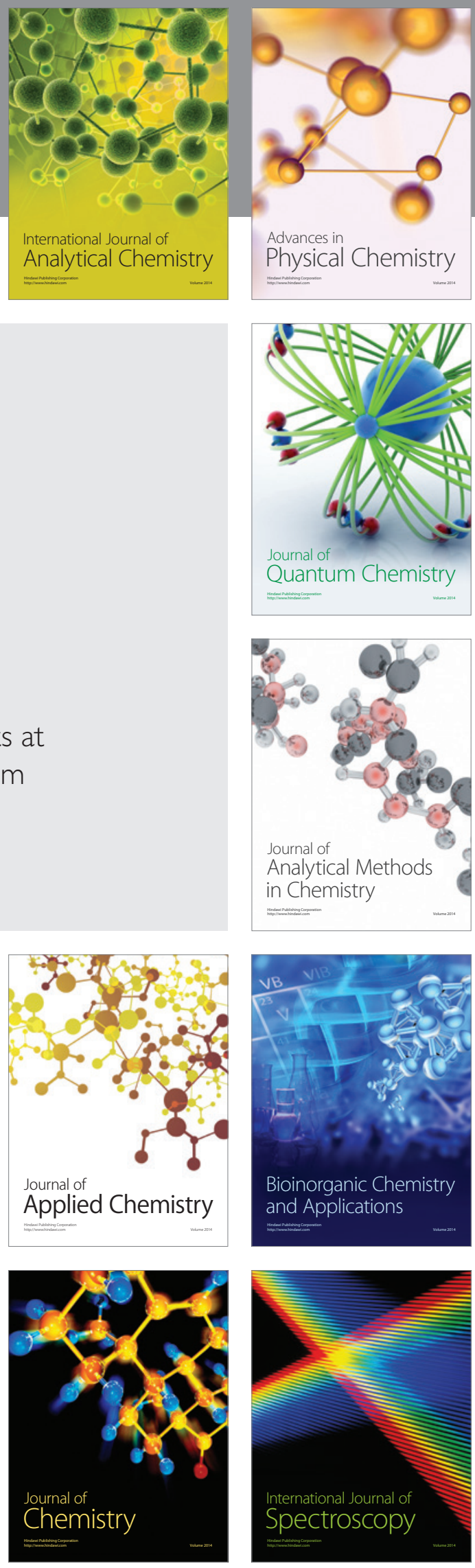